\title{
Impact of ivermectin mass distribution for onchocerciasis control on Ascaris lumbricoides among schoolchildren in Rungwe and Kyela Districts, southwest Tanzania
}

\author{
B.T.A. MAEGGA ${ }^{*}$, K.D. MALLEY and V. MWIWULA ${ }^{\dagger}$ \\ National Institute for Medical Research, Tukuyu Medical Research Station \\ P. O. Box 538, Tukuyu, Tanzania
}

\begin{abstract}
A cross-sectional survey on the impact of ivermectin treatment for onchocerciasis control, on Ascaris lumbricoides infections was conducted in 1994, before the first treatment cycle was implemented in the Tukuyu focus in Tanzania. A total of 560 pupils were randomly selected for stool samples before, 7 days and 3 months after treatment. Modified Kato Katz technique was used to prepare slides, and a dissection microscope for examination. Results showed pre-treatment A. lumbricoides prevalence ranging from $4.2 \%$ (Lutete) to $34.0 \%$ (Lufilyo). At day seven post-treatment, samples were devoid of Ascaris eggs, except at Lufilyo with 2.9\% prevalence. Three months post treatment showed $34(8.2 \%)$ out of 213 pupils had patent infections and $24(5.8 \%)$ re-infections. Mean infection intensities were significantly higher at pretreatment than three months post-treatment. A. lumbricoides adult worm expulsion was self-reported in the first week of treatment, especially 2-3 days after, by $21.9 \%$ participants. Ivermectin treatment was effective against adult $A$. lumbricoides and cleared egg excretion by day seven, but re-infection occurred within three months. Visible worm excretion was appreciated as potency of treatment. Long term mass treatment with ivermectin proposed for onchocerciasis control can be used to reduce $A$. lumbircoides infections. To benefit optimally from this programme, additional inputs should be availed to reduce re-infection between consecutive annual cycles. Appreciation of visible worm expulsion enhanced acceptance and compliance of onchocerciasis control campaign using ivermectin. Follow-up surveys are recommended.
\end{abstract}

Keywords: Ivermectin, onchocerciasis, Ascaris lumbricoides, schoolchildren, Tanzania

\section{Introduction}

Ivermectin is a semi-synthetic macrolide lactone which has entirely transformed strategies for the treatment and control of onchocerciasis and lymphatic filariasis since its registration for human use in 1987 (Oliver, 1994). In addition, the drug has significant effects on a wide range of other helminths and ectoparasites (Shastri, 1991; Meinking et al., 1995; Heukelbach et al., 2004). Various clinical trials have shown that a single dose of ivermectin given once a year leads to a marked reduction in skin microfilaria counts of Onchocerca volvulus to undetectable levels for several days (Taylor et al., 1990), with a long lasting suppressive effect (Schulz-Key et al., 1992; Cupp et al., 1986).

The effectiveness of ivermectin against soiltransmitted helminths such as Ascaris lumbricoides, Trichuris trichiura and hookworms in other parts of the world has been reported by Cupp (1992) and Njoo et al. (1990). In Tanzania, Makunde et al. (1991) reported similar observations with $A$. lumbricoides in the Eastern Usambaras, but, the drug did not seem to be effective against concomitant hookworm infections.
Human infections by soil transmitted helminths such as A. lumbricoides are very common in Rungwe and Kyela Districts in south-west Tanzania, where annual rainfall is above $2,000 \mathrm{~mm}$ (Atlas of Tanzania, 2nd Edition). Until very recently, the normal status of community sanitary facilities was rather poor in rural areas, thus encouraging contamination of soil. Although recent literature on the prevalence and intensities of these parasitic infections is hard to come by, anecdotal evidence from enlightened residents who resort to deworming their children and pets like cats and dogs, indicates intense transmission of these infections. Being a large parasitic worm, $A$. lumbricoides infections are easily recognisable by all affected, who confess of having "snakes in their stomachs", and during the initial round of ivermectin administration, many persons came back with their family members asking for more tablets to clean their bodies of the parasites.

A large scale community based treatment of onchocerciasis using ivermectin was initiated in the Tukuyu onchocercaisis focus in mid 1994, and was anticipated to continue for up to 20 years. In most of the onchocerciasis endemic countries in Africa,

\footnotetext{
* Correspondence: Dr. Bertha T.A. Maegga; E-mail: maeggabta@africaonline.net

t Deceased
} 
ivermectin mass distribution is done once every year, to cover all persons from 5 years and above, in all communities with disease prevalence of $20 \%$ and above, derived from nodule rate surveys. This strategy presumes that, within the 15 to 20 year period, the adult worm population in the treated communities will die out from the natural ageing process, and transmission reduction through attrition over these many years would have prevented new infections in areas under treatment.

A cross sectional study on the impact of ivermectin treatment on $A$. lumbricoides was undertaken in an onchocerciasis endemic area. The objective of the study was to obtain baseline data on the prevalence and intensity of $A$. lumbricoides infections in the onchocerciasis endemic communities and assess the effectiveness of ivermectin on this helminth species.

\section{Materials and Methods}

\section{Study area}

The study was conducted in Rungwe and Kyela districts of Mbeya region, southwest Tanzania, in an onchocerciasis endemic focus which was about to start mass distribution of ivermectin. The onchocerciasis focus was estimated to fall within the area of about $3,000 \mathrm{~km}^{2}$. It lies between $9^{\circ} 5^{\prime} \mathrm{S}$ and $9^{\circ} 45^{\prime} \mathrm{S}$ and $33^{\circ} 20^{\prime} \mathrm{E}$ and $34^{\circ} 20^{\prime} \mathrm{E}$. It is a densely populated area, with an average density of 69.4 persons per $\mathrm{km}^{2}$. The area is mountainous, and lies within the Great Rift Valley, whose two main branches meet just north of Lake Nyasa, at an altitude of $480 \mathrm{~m}$, while the volcanic summits of Rungwe and Kyejo rise to altitudes of over 2,000m.

The climate is favourable, rainfall coming from November to May / June, and dry cool season from July to October. There is a spell of less rain in January and February, dividing the long rains of March to May, and the short rains of November and December. The annual mean rainfall usually exceeds 2,000mm.

Five primary schools, in areas covered by the Onchocerciasis Control Project area, were selected for the study. The schools lie at different altitudinal ranges, and included: Lufilyo and Kibole in higher altitudes (650 to $750 \mathrm{~m}$ ), Kisegese and Lwangwa- Masoko in lower altitudes (500$590 \mathrm{~m}$ ) and Lutete village is partly on high ridge and partly in the valley of Kiwira River, both falling within either of the above two categories.

\section{Study design}

This was a cross sectional study that was carried out just before ivermectin mass distribution to control onchocerciasis. School selection was dictated by altitudinal variation and whether they were on hill slopes or valley and lowland flood plains. The sampling was done in primary schools for easy management, to represent their communities where onchocerciasis was highly endemic and under proposed ivermectin mass treatment. Schoolchildren were registered and those who provided stool specimens were randomly selected from the registered list. Children from whom samples were requested were from class I to VI (range 8 to 16 years).

The first stool specimens were taken on the same day of drug taking, but before it were swallowed. The survey team visited each school a day before drug distribution, and conducted advocacy, mobilization/sensitization and registration of children. Out of 1,146 schoolchildren registered, $560(48.9 \%)$ were selected and requested to give stool specimens. A day before specimen collection, plastic cups were distributed to children which were randomly selected from those registered. Stool specimens were then collected the following morning. These were labelled and transported immediately to the nearby health facility for examination, and slide preparation using modified Kato Katz technique for later microscopy. Slides were later examined for the presence of $A$. lumbricoides. Reports of worm discharge were given to teachers, who recorded voluntary information from participants at four schools, who reported their experiences within the treatment week.

At 7 days post-treatment, samples were obtained from those pupils who were positive for A. lumbricoides the first time. Approximately three months later the initial pupil sample was again requested to supply stool specimens, which were handled in the same manner.

\section{Mobilization, sensitization and ethical consideration}

Prior to the surveys, letters giving permission to conduct the study in schools were obtained from the respective district educational authorities, and the same were distributed to the head-teachers of the selected schools. Sensitization and mobilization was done through the school administration, and the school head teachers were asked permission of sampling stools. Oral consent was also sought and obtained from schoolchildren, to donate stool samples before they took Ivermectin and afterwards, at 7 days and 3 months post treatment.

\section{Results}

Of the 560 pupils examined at pre-treatment, the prevalence of A. lumbricoides was $34.0 \%$ at Lufilyo, $13.0 \%$ at Kibole, $13.1 \%$ at Lwangwa-Masoko, $10 \%$ at Kisegese and $4.2 \%$ at Lutete (Table 1). At seven days after treatment, four schools reported no detectable 
A. lumbricoides infections in the previously infected pupils, but Lufilyo had $2.9 \%$.

After three months, results showed that the overall prevalence of $A$. lumbricoides was $14.6 \%$. The pre-treatment mean infection per school was greater than three months post-treatment (expressed in eggs per gm of faeces) in each of the five primary schools. The number and percentage of children who reported adult worm expulsion after taking a single dose of ivermectin from four schools is summarised in Table 2.
Records from Kibole school were not available. Newly acquired infections and re-infections of previously infected and cleared A. lumbricoides among the schoolchildren during the posttreatment follow up approximately three months later is shown in Table 3. Generally, A. lumbricoides prevalence was higher in schools located in the higher altitudes.

Table 1: Pre-treatment prevalence of Ascaris lumbricoides in five primary schools

\begin{tabular}{lllll}
\hline Name of school & $\begin{array}{l}\text { No. pupils } \\
\text { registered }\end{array}$ & $\begin{array}{l}\text { No. pupils } \\
\text { examined }\end{array}$ & $\begin{array}{l}\text { No. pupils with } \\
\text { A. lumbricoides }\end{array}$ & Prevalence (\%) \\
\hline Lufilyo & 264 & 100 & 34 & 34.0 \\
Kibole & 95 & 90 & 12 & 13.0 \\
Kisegese & 135 & 120 & 12 & 10.0 \\
Lutete & 412 & 120 & 5 & 4.2 \\
Lwanga-Masoko & 240 & 130 & 18 & 13.1 \\
Total & 1146 & 560 & 81 & 14.5 \\
\hline
\end{tabular}

Table 2: Schoolchildren reporting adult worm expulsions after a single dose of ivermectin

\begin{tabular}{lccc}
\hline School & No. registered & $\begin{array}{c}\text { No. reporting worm } \\
\text { expulsions }\end{array}$ & $\%$ \\
\hline Lufilyo & 264 & 88 & 33.5 \\
Kibole & 95 & 21 & 22.1 \\
Lutete & 412 & 71 & 17.2 \\
Kisegese & 135 & NR & NR \\
L/Masoko & 240 & 41 & 17.1 \\
Total & 1146 & 221 & 21.9 \\
Key: NR= No Record & & & \\
\hline
\end{tabular}

Table 3: Ascaris lumbricoides infection status in schoolchildren 3 months post-treatment

\begin{tabular}{llcccccc}
\hline School & $\begin{array}{l}\text { No. pupils } \\
\text { examined }\end{array}$ & \#A & $\begin{array}{l}\text { Prevalence } \\
(\%)\end{array}$ & \#B & $\begin{array}{l}\text { Prevalence } \\
(\%)\end{array}$ & $\begin{array}{l}\text { \#A+B } \\
\text { Prevalence } \\
(\%)\end{array}$ \\
\hline Lufilyo & 31 & 0 & 0.0 & 3 & 9.7 & 3 & 9.7 \\
Kibole & 76 & 3 & 4.0 & 2 & 2.6 & 5 & 6.6 \\
Kisegese & 93 & 4 & 4.3 & 5 & 5.4 & 9 & 9.7 \\
Lutete & 98 & 7 & 7.1 & 1 & 1.0 & 8 & 8.2 \\
Lwanga-Masoko & 115 & 20 & 17.4 & 13 & 11.3 & 33 & 28.7 \\
Total & 413 & 34 & 8.2 & 24 & 5.8 & 58 & 14.0
\end{tabular}

Key: $\quad \mathbf{A}=$ Those pupils initially negative for A. lumbricoides infection but treated to expel any young worms which may not have laid eggs.

$\mathbf{B}=$ Those pupils initially positive for A. lumbricoides but were negative at day seven post-treatment. The reappearance of Ascaris eggs after treatment was considered as re-infection 


\section{Discussion}

The results of this study further confirm observations made by other workers in various geographical regions that a single low dose of ivermectin administered orally, effectively reduces prevalence and intensity of $A$. lumbricoides infections (Heukelbach et al., 2004). The observations made within the seven days posttreatment include self-reported adult worm expulsion by children who swallowed ivermectin. Stool examination after seven days of treatment showed that most of those initially positive for $A$. lumbricoides infections were cleared. At this stage, the negative observations in the previously positive children were a clear indication of complete parasitological clearance of Ascaris eggs, more probably due to adult worm death and excretion.

The low positive percentage found at Lufilyo school at day seven could have been a treatment failure. However, the pupils were not appropriately followed-up, so the cause could not be established. On the other hand, the observations after three months showed a considerable reinfection, which must have occurred after the treatment date.

Since ivermectin treatment for onchocerciasis control is taken once per year, it is certain that, A. lumbricoides re-infection will occur way before a subsequent dose is taken. The current observations showed substantial re-infection at three months, and will no doubt, continue to increase with time, prior to the subsequent treatment date. On the other hand, at three months, the intensity of infection had not yet reached the pre-treatment levels, and thus, if there is any respite for those who are liable to worm infection, they will most likely have lower infection intensity, and thus less worm burden, even if the prevalence may rise to pre-treatment levels. It will be interesting to follow up what happens with repeated ivermectin treatment every year, for the duration of active onchocerciasis control programme in the Tukuyu focus. This may offer a window of opportunity to launch community based or community directed worm control campaign in the area, to synergise with the ongoing community directed treatment with ivermectin.

Community members in the area recognise worm infections as a significant problem. Hence the study participants were very willing to voluntarily report their experience, recording the number of worms they excreted. During the first ivermectin treatment campaign, many adult persons also reported worm expulsion, and visited the study team demanding for more tablets. The tangible effects of ivermectin through Ascaris worm discharge were one of the positive factors that enhanced ivermectin mass drug administration programme acceptance.

The baseline information obtained is evidence of effectiveness of the drug on $A$. lumbricoides, and also showed that re-infection occurred fairly fast. This information can be used to plan treatment schedules for deworming, and in the onchocerciasis control areas, to recommend appropriate measures for setting up addition inputs so that the communities can further benefit in a deworming programme pegged onto the set up national programme for onchocerciasis control where this is in place, and where A. lumbricoides is co-endemic with onchocerciasis.

In view of the observed rates of infection and re-infection among the surveyed primary schools, it is proposed that for any effective control of ascariasis in the area, regular de-worming of the whole communities is necessary, and that to be done at intervals of less than three months to reduce re-infection frequencies by attrition. After the initial worm clearance, the intensity of infection at three months was lighter, thus repeated treatments should progressively reduce the burden of disease resulting from heavy infection. Due to contamination of the environment, intense advocacy on health education focusing on fruit and vegetable sanitation as well as personal and public hygiene are recommended. Proper disposal of human waste in the communities including primary schools and public places should be enforced in order to enhance environmental decontamination, subsequently reducing soil incubating worms in the area.

In conclusion, ivermectin is effective in clearing A. lumbricoides, resulting in extensive adult worm expulsion and clearing egg excretion in stools, within a week of a single dose. Considerable re-infection of $A$. lumbricoides resulting in patent infections occurred within three months of a single treatment. Similar findings have been reported elsewhere (Shield et al., 1984; Luoba et al., 2005). Visible worm expulsion was appreciated by study participants, who asked for more tablets. This greatly benefited in compliance to treatment schedule in the initial stages of onchocerciasis control campaign.

Onchocerciasis control using large scale ivermectin administration is anticipated to last for 20 years, this opportunity should be used to reduce A. lumbricoides infections in the area covered by the programme, and follow-up studies are recommended in order to document its contribution. To benefit optimally from the onchocerciasis control programme, it is recommended that responsible institutions make available additional inputs in order to reduce reinfection rates between consecutive single annual cycles of ivermectin, as it is now set up. 


\section{Acknowledgements}

We are grateful to Merck, Sharp and Dohme for free donation of ivermectin, and the River Blindness Foundation of Sugar Land, Texas, U.S.A. for logistical support that facilitated its distribution in the primary schools studied. We further appreciate the material support of Ifakara Health Research and Development Centre for Kato Katz laboratory technique. We gratefully appreciate the corporation of the District Education Authorities and the head teachers for allowing the study to be executed in their premises. Our special thanks go to the schoolchildren and their parents and guardians for their willingness not only to participate but also for their ceaseless cooperation during the execution period. We are greatly indebted to the Ministry of Health and the National Institute for Medical Research for making available the funds obtained through the EU-Financial grants system.

\section{References}

Brown, K.R. \& Nay, D.C. (1990) Ivermectin Clinical trials and treatment schedules in onchocerciasis. Acta Leiden 59, 169-175.

Cupp, E.W. (1992) The treatment of Onchocerciasis with ivermectin in Central America. Parasitology Today 8, 212-214.

Cupp, E.W., Bernado, M.J., Kiszewski, A.E., Collins, Taylor, H.R., Azizi, M.A. \& Green, B.M. (1986) The effects of ivermectin on transmission of Ochocerca volvulus. Science 231, 740-742.

Heukelbach, J., Winter, B., Wilcke, T., Muehlen, M., Albrecht, S., de Oliveira, F.A.S., Kerr-Pontes, L.R.S., Liesenfeld, O. \& Feldmeier, H. (2004) Selective mass treatment with ivermectin to control intestinal helminthiases and parasitic skin diseases in a severely affected population. Bulletin of the World Health Organization 82, 563-571. I
Luoba, A.I., Wenzel Geissler, P., Estambale, B., Ouma, J.H., Alusala, D., Aya, R., Mwaniki, D., Magnusen, P. \& Friis, H. (2005) Eartheating and re-infection with intestinal helminths among pregnant and lactating women in western Kenya. Tropical Medicine and International Health 10, 220-227.

Makunde, W.H., Msangeni, H.A., Irare, S.G.M. \& Massaga, J.J. (1991) Evaluation of the efficacy of ivermectin in the treatment of intestinal helminths and urinary trematoda at Amani, Tanzania. Annual Report of the National Institute for Medical Research 10, 35-36.

, T.L., Taplin, D., Herminda, J.L., Pardo, R. \& Kerdel, F.A. (1995) The treatment of scabies with ivermectin. New England Journal of Medicine 333, 26-30.

Njoo, F.L., Belling, G.A.C., Oosting, J. Vettec J.C.M. Stilma, J.S. \& Kijlstra (1993) Concurrent parasitic infections in onchocerciasis and the occurrence of adverse reaction after ivermectin treatment. American Journal of Tropical Medicine and Hygiene 48, 652-657.

Oliver C.W. (1994) Talking Drug: A Communication Handbook for field managers of Riverblindness Prevention Programs. Pg. 18.

Schulz-Key, H., Soboslay, P.T. \& Hoffman W.H. (1992) Ivermectin facilitated immunity. Parasitology Today 74,194-199.

Shastri, U.V. (1991) Efficacy of ivermectin (Msd) against lice infestation in cattle, buffalos, goats and dogs. Indian Veterinary Journal 68, 191.

Shield, J., Anian, G., Ostwald, R \& Arnhold, R. (1984) Re-infection with intestinal helminths after treatment with mebendazole and fluctuations in individual Ascaris lumbricoides with time. Papua New Guinea Medical Journal 27, 89-94.

Taylor, H.R., Pacque, M., Munoz, B. \& Green, B.M. (1990) Impact of mass treatment of onchocerciasis with ivermectin on transmission of infection. Science 250, 115-118. 